\title{
Comparison of the Thermal and Electrical Conductivities of Pretreated Kenaf-Polyester Composites
}

\author{
P. U. N. Obianuko, J. C. Obianuko, and T. U. Onuegbu
}

\begin{abstract}
Conductivity of a material is an important physical property that determines its suitability or otherwise in all engineering designs and construction. The aim of this study is to determine the effects of two pretreatment methods viz acetylation and permanganate on the thermal and electrical conductivities of pretreated kenaf bast fibres applied in polyester resin. Fully grown kenaf (hibiscus cannabalis) were manually retted from the stalk, washed, and cut into short fibre lengths of about $10 \mathrm{~cm}$. One portion of the fibres was pretreated with $5 \%$ pbw $\mathrm{NaOH}$ solution before immersing it in glacial acetic acid and then in acetic anhydride. The second was also pretreated with $5 \%$ pbw of $\mathrm{NaOH}$ before being pretreated with $0.125 \%$ of $\mathrm{KMnO}_{4}$. The third portion of fibres was untreated to serve as control. The ground fibre was incorporated into ortho unsaturated polyester rand cast with moulds of dumb-belland square shapes. The electrical conductivity of the composites was deduced by measuring the resistance of the composites using the high voltage insulation tester model 3122 and calculating from equations. The thermal conductivities were determined by analytical method. The results show that chemical pretreatments of fibres by acetylation and permanganate methods have no appreciable effect on the thermal conductivities of composites. Further findings show that the acetylated fibre composites have no effect on the electrical conductivities of the composites. The permanganate pretreated fibre composites however increased the electrical conductivities of the composites significantly.
\end{abstract}

Keywords - composites, conductivities, fibre, preatreatment.

\section{INTRODUCTION}

Natural fibre composites are increasingly being applied in non-structural application of engineering designs in preference to synthetic fibre composites. The obvious reason for this is because of some disadvantages of synthetic fibres which include environmental pollution due to the nondegradability of fibres and difficulties for these fibres to be recycled, and non-sustainability especially with continuous depletion of petroleum resources [1]. Natural or green fibre composites also have their own problems. These include poor wettability, incompatibility with some polymeric matrices and high moisture absorption due to their hydrophilic property. Composite materials made with unmodified plant fibres frequently exhibit unsatisfactory mechanical properties [2]. These disadvantages have sparked of research interest in the area of surface modification of fibres by chemical pretreatments. One of the major chemical treatments is coupling which includes silane coupling,

Submitted on May 11, 2021.

Published on September 28, 2021.

P. U. N. Obianuko, Raw Materials Research and Development Council, Nigeria.

(e-mail: ucheobianuko@yahoo.com) acetylation, malleated polymer and isocyanate. Using epoxy and unsaturated polyester resins it has been shown that fibre treatment with silane coupling agent bearing functional group, which was able to react with matrix, enhanced significantly the mechanical strength of the final composite [3]. In another of such studies by [4], acrylic acid was used as the coupling agent while in yet another study [5] MAPP was used as coupling agent in jute. It was also reported in another work [6] that coupling with maleic anhydride treatment reduced water absorption to a great extent in hemp, banana, and sisal fibres and their composites. It is important to ensure that chemical pretreatment does not introduce undesirable properties in the composites. For instance, a recent study by [7] reported that chemical modification resulted in an increase of $43 \%$ in thermal conductivity when compared with untreated fibre-composite. This may not be a property to be desired since most polymer composites are used as casings and the interiors of motor vehicles where there insulating properties is a major criterion for use. It is therefore important to investigate the effects of these two chemical pretreatment methods on the thermal and electrical conductivities of composites of pretreated kenaf-polyester composites.

\section{EXPERIMENTAL}

\section{A. Chemical Pretreatment of Fibres}

The prepared kenaf bast fibres were cut into short length fibres of about $10 \mathrm{~cm}$ and divided into three separate portions as follows: One portion was chemically pretreated with potassium permanganate; known as permanganate pretreatment. The second portion was pretreated with glacial acetic acid and acetic anhydride; known as acetylation. The third portion of fibres was untreated to serve as control.

\section{1) Permanganate Pretreatment of Kenaf Fibres}

The treatment with potassium permanganate was preceded by dilute alkaline treatment. This was to create active reaction sites in the fibres.

\section{1) Procedure}

$50 \mathrm{~g}$ of sodium hydroxide pellets $\left(\mathrm{NaOH}_{(\mathrm{s})}\right)$ was weighed and dissolved in $950 \mathrm{~g}$ of water to prepare $5 \%$ parts by weight $(p b w)$ of $\mathrm{NaOH}_{\text {aq }}$. The solution was stirred gently until all the pellets had completely dissolved. One portion of the fibre was immersed in the $5 \% p b w$ of $\mathrm{NaOH}_{\mathrm{aq}}$ and pressed gently to ensure complete immersion of the fibres. It was allowed to stand for 30 minutes, then the fibre was removed and allowed

J. C. Obianuko, Department of Agric Engineering, Kaduna Polytechnic Kaduna, Nigeria.

T. U. Onuegbu, Department of Industrial Chemistry, Nnamdi Azikiwe University, Awka, Nigeria. 
to drain. $0.125 \%$ pbw of potassium permanganate solution was prepared by dissolving $1.25 \mathrm{~g}$ of $\mathrm{KMnO}_{4}$ crystals in $998.75 \mathrm{~g}$ of acetone [8]. The alkaline conditioned fibres were then immersed in the potassium permanganate in acetone for three minutes ( 3 mins). The fibre was thereafter removed and drained in the sieve and dried in the Gallenkamp oven at a temperature of $105^{\circ} \mathrm{C}$ until it was crisp dry.

\section{2) Acetylation Pretreatment}

As was the case in permanganate pretreatment, the second portion of fibre sample was treated with alkaline by immersing it in $5 \%$ pbw of sodium hydroxide solution for 30 minutes, removed and allowed to drain thoroughly. In the acetylation process the fibre was first immersed in glacial acetic acid for one hour. The glacial acetic acid was decanted and the fibre was finally immersed in acetic anhydride with two drops of conc $\mathrm{H}_{2} \mathrm{SO}_{4}$ for five minutes [9].

The fibre was then removed and drained in the sieve and allowed to dry. The acetylated fibre was further dried in the oven at temperature of $105{ }^{\circ} \mathrm{C}$ until it was crisp dry. The third sample which was untreated was also dried under the same conditions of temperature as the pretreated samples. All the fibre samples were packaged in airtight containers. The separate fibre samples were milled into rough fibre particles and stored in airtight containers.

\section{2) Preparation of Polyester Composite}

The mould was first cleaned with cotton wool dipped in acetone to remove dirt and was allowed to dry. Poly Vinyl Alcohol $\left(\mathrm{PVA}_{(\mathrm{l})}\right)$ was then applied uniformly on the surface of the mould with a short wooden spatula. A thin film of $\mathrm{PVA}_{(\mathrm{s})}$ formed on the mould when the PVA is dried acts as the mould releasing agent. (Note that the aluminum mould was dismantled to ensure uniform coating of the mould surface and fixed again after it was dried). The fibre sample and polyester were weighed using the electronic balance. The fibre was mixed with the polyester at room temperature and stirred continuously for 3 minutes until a homogenous mixture was observed. $2 \%$ (by weight of polyester) of the catalyst, methyl ethyl ketone peroxide (MEKP) was added using the syringe and stirred continuously for another 3 minutes. Finally, $1 \%$ (by weight of polyester) of the accelerator; cobalt octoate was added and stirred for another 3 minutes. The reaction temperature was taken, and the composite was cast in the moulds and allowed to cure for one hour. The cured samples were removed from the mould and the overflow flakes were cut off using the small plier. The procedure was repeated for all the fibre volume fraction of $0.04,0.08,0.12$ and 0.16 and for each sample of fibre viz: the permanganate treated fibre, the acetylated fibre and the untreated fibre. To study the effect of the fibre reinforcement, the unreinforced (zero fibre volume) sample of the polyester was also prepared.

The formulation for the fibre-ortho unsaturated polyester composite is as shown in the table below.

TABLE I: FoRMULATION OF FIBRE-ORTHO UNSATURATED POLYESTER COMPOSITE

\begin{tabular}{ccccc}
\hline Reagents & \multicolumn{4}{c}{ Weight in Grammes } \\
\hline Parts by weight of & $(4 \%)$ & $(8 \%)$ & $(12 \%)$ & $(16 \%)$ \\
\hline
\end{tabular}

\begin{tabular}{ccccc}
\hline Ground Kenaf & $4 \mathrm{~g}$ & $8 \mathrm{~g}$ & $12.0 \mathrm{~g}$ & $16.0 \mathrm{~g}$ \\
$\begin{array}{c}\text { Ortho unsaturated polyester } \\
\text { Methyl Ethyl Ketone }\end{array}$ & $96.0 \mathrm{~g}$ & $92.0 \mathrm{~g}$ & $88.0 \mathrm{~g}$ & $84.0 \mathrm{~g}$ \\
$\begin{array}{c}\text { Peroxide (MEKP) (2\% } \\
\quad \text { weight of polyester) }\end{array}$ & 1.92 & 1.84 & 1.76 & 1.64 \\
$\begin{array}{c}\text { Accelerator-Cobalt Octoate } \\
\text { (1\% weight of polyester) }\end{array}$ & 0.96 & 0.92 & 0.88 & 0.84 \\
Reaction Temperature in ${ }^{\circ} \mathrm{C}$ & 44 & 42 & 40 & 40 \\
$\quad$ Curing Temperature & 45 & 43 & 42 & 42 \\
\hline
\end{tabular}

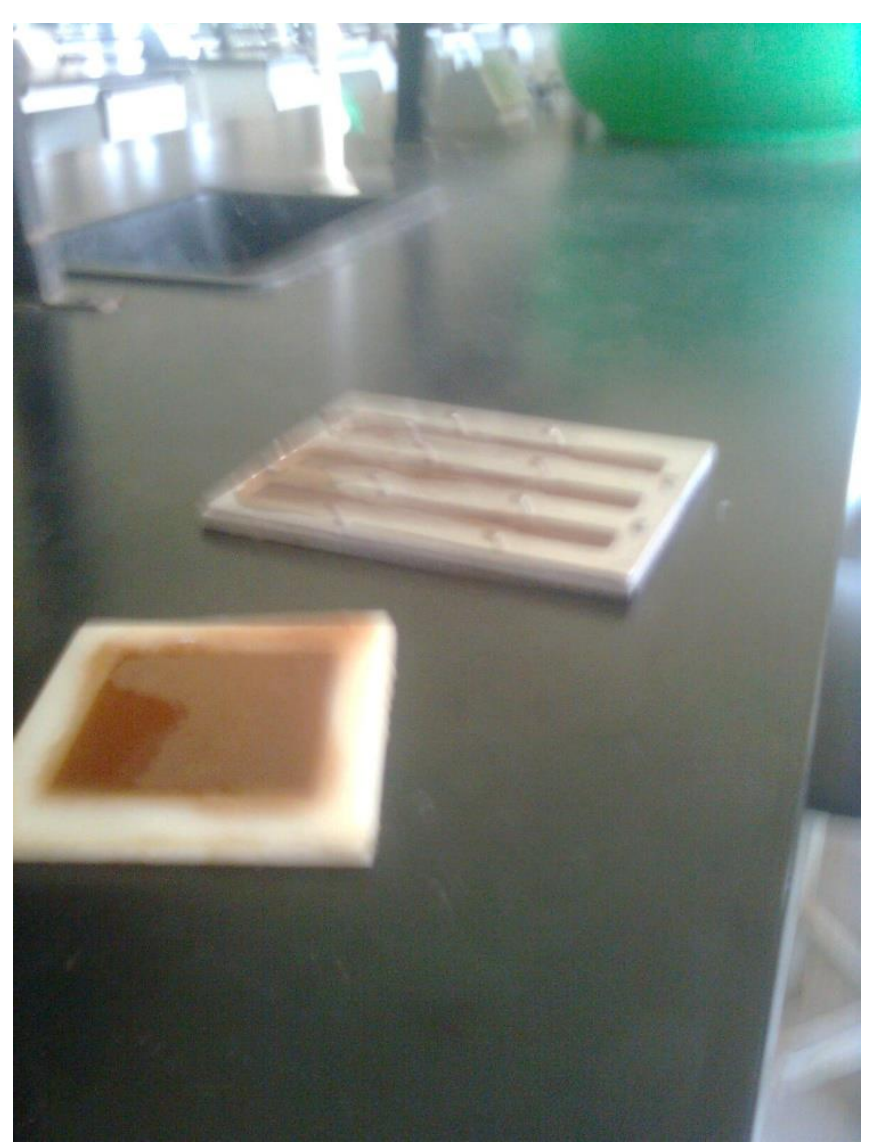

PLATE 1. Composite Samples Curing in the mould.

\section{B. Thermal Conductivities}

The thermal conductivities were determined by analytical method. The composite samples were cut into small samples of measured dimensions (length, width, and thickness) and weighed. They were heated in turn in an oven of $1 \mathrm{Kw}$ (1000 wts) for one hour (60 min). The samples were preconditioned to assume the room temperature as the initial temperature. After heating for one hour, the final temperature of the sample was determined using the thermocouple:

$$
\begin{aligned}
& H=\frac{\Delta Q}{\Delta t} \\
& k A=\frac{\Delta T}{X t} \mathrm{kA} \\
& \frac{\Delta Q}{\Delta t}=\text { rate of heat flow } \\
& K=\frac{\Delta Q}{\Delta t} \times \frac{1}{A} \times \frac{X t}{\Delta T}
\end{aligned}
$$

$A=L \times W$ of the material;

$X_{t}=$ thickness of material;

$\Delta T=$ Temperature difference; $t=$ Time; 
$\Delta Q=$ Quantity of heat $=m c \Delta T$ (c is specific heat capacity $).$

$$
\begin{aligned}
& P t=m c \Delta T, c=\frac{P t}{m \Delta T} \\
& K=\frac{m c \Delta T}{t} \times \frac{1}{A} \times \frac{X t}{\Delta t}
\end{aligned}
$$

Therefore,

$$
K=\frac{M P t \Delta T}{t \times m \times \Delta T} \times \frac{1}{A} \times \frac{X t}{\Delta t}
$$

Cancelling out like terms, we have:

$$
K=P \times \frac{1}{A} \times \frac{X t}{\Delta t}
$$

Using the above relationship in (7), $\mathrm{K}$ is determined from the data on page 3 .

\section{Electrical Conductivities}

Electrical conductivity is the inverse of electrical resistivity:

$$
\text { Electrical conductivity }=\frac{1}{\rho}
$$

Resistivity is related to electrical resistance by the following equation:

$$
\rho=\frac{R \cdot A}{L}
$$

Therefore, $G$, electrical conductivity, is given by the equation:

$$
G=\frac{L}{R \cdot A}
$$

where $L$ is length of material, $R$ is resistance and $A$ is cross sectional area of the material. The electrical conductivity is deduced from the above equation by measuring the resistance of the material using the high voltage insulation tester model 3122. The length and width of the material are measured with transparent ruler and hence the cross-sectional area is deduced.

\section{PResentation AND Analysis Of Results}

\section{A. Thermal Conductivities of Composites}

The Fig. 1 below shows that the thermal conductivities of the composites show no significant difference in their thermal conductivities for both the treated and untreated fibres. This is also true when the reinforced polyester composites are compared with the unreinforced polyester. The reinforcement with fibre or its pretreatment did not increase the thermal conductivities of the polyester composites significantly.
TABLE II: THERMAL CONDUCTIVITIES OF FIBRE-ORTHO UNSATURATED POLYESTER COMPOSITE (W/MK)

\begin{tabular}{ccccc}
\hline Materials & Mass $(\mathrm{g})$ & $\begin{array}{c}\text { Temperature } \\
\text { change } \Delta \mathrm{T} \\
(\mathrm{K})\end{array}$ & Area $\left(\mathrm{mm}^{2}\right)$ & $\begin{array}{c}\text { Thermal } \\
\text { Conductivity } \\
(\mathrm{W} / \mathrm{mK})\end{array}$ \\
\hline ATS 04\% & 1.76 & 25 & 731.0 & 0.201 \\
ATS 08\% & 3.81 & 23 & 760.0 & 0.200 \\
ATS 12\% & 5.68 & 27 & 760.0 & 0.167 \\
ATS 16\% & 3.92 & 18 & 741.0 & 0.274 \\
PTS 04\% & 1.68 & 25 & 798.0 & 0.185 \\
PTS 08\% & 1.87 & 23 & 779.0 & 0.195 \\
PTS 12\% & 3.77 & 23 & 807.5 & 0.189 \\
PTS 16\% & 3.79 & 23 & 731.5 & 0.208 \\
STS 0\% & 3.64 & 23 & 795.5 & 0.191 \\
UTS 04\% & 9.38 & 21 & 817.0 & 0.217 \\
UTS 08\% & 7.86 & 29 & 798.0 & 0.159 \\
UTS 12\% & 3.57 & 23 & 798.0 & 0.190 \\
UTS 16\% & 3.85 & 18 & 826.5 & 0.246 \\
\hline
\end{tabular}

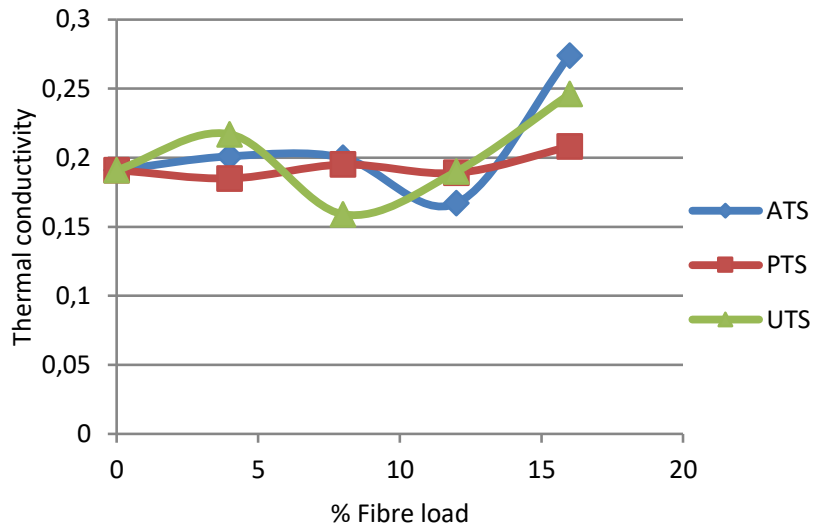

Fig. 1. Thermal Conductivities of Composites.

B. Electrical Conductivities of Composites

TABLE III: ELECTRICAL CONDUCTIVITIES OF FIBRE POLYESTER COMPOSITES (S/M)

\begin{tabular}{ccccc}
\hline Materials & $\begin{array}{c}\text { Resistance } \\
(\mathrm{M} \Omega) \times 1000\end{array}$ & Area $\left(\mathrm{m}^{2}\right)$ & Length $(\mathrm{m})$ & $\begin{array}{c}\text { Electrical } \\
\text { Conductivity } \\
(\mathrm{S} / \mathrm{m})\end{array}$ \\
\hline ATS 04\% & 300,000 & 0.000817 & 0.0430 & 0.00018 \\
ATS 08\% & 110,000 & 0.000850 & 0.0425 & 0.000046 \\
ATS 12\% & 500,000 & 0.000779 & 0.0410 & 0.00011 \\
ATS 16\% & 267,000 & 0.0008075 & 0.0425 & 0.00019 \\
PTS 04\% & 40,000 & 0.000860 & 0.0430 & 0.0013 \\
PTS 08\% & 30,000 & 0.000760 & 0.0400 & 0.0018 \\
PTS 12\% & 50,000 & 0.000740 & 0.0400 & 0.0011 \\
PTS 16\% & 20,000 & 0.0007695 & 0.0405 & 0.0026 \\
STS 0\% & $200,000,000$ & 0.000798 & 0.0420 & 0.00000026 \\
UTS 04\% & $180,000,000$ & 0.000741 & 0.0390 & 0.00000029 \\
UTS 08\% & $80,000,000$ & 0.0008075 & 0.0425 & 0.00000066 \\
UTS 12\% & 600,000 & 0.000798 & 0.0420 & 0.000088 \\
UTS 16\% & $16,000,000$ & 0.000760 & 0.0400 & 0.0000033 \\
\hline
\end{tabular}

The results of the electrical conductivities as shown in the Fig. 2 and Table III show that the composites of the acetylated fibre and untreated fibre have poor electrical conductivity. The high values of the resistances measured indicate that conduction of electricity is almost impossible. However, the graph of the electrical conductivities of the permanganate treated fibre-composite increased significantly as fibre volume fraction increased by more than $100 \%$. Permanganate pretreatment may therefore be useful in compounding electrical conducting polymer composites. 


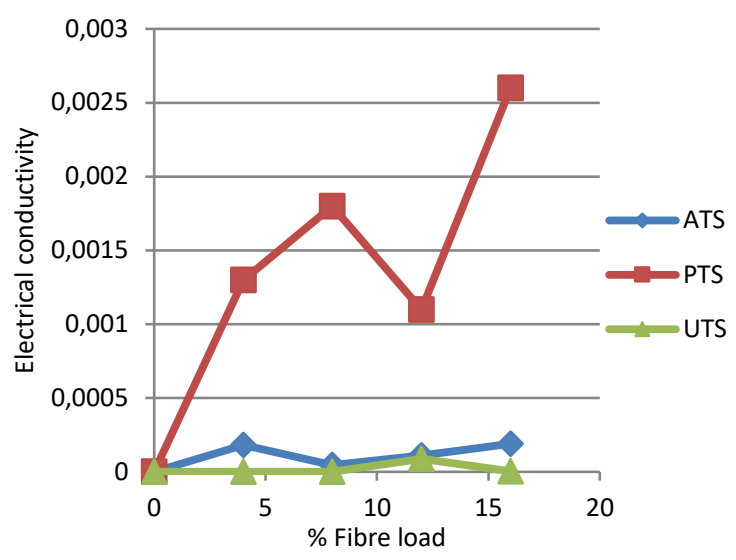

Fig. 2. Electrical Conductivities of Composites.

\section{CONCLUSION}

The obvious conclusion drawn from this work is that acetylation of kenaf bast fibres used in $\mathrm{p}$ resin to produce composites have no effect on the thermal and electrical conductivities of the resulting composites. Permanganate pretreatment of the fibres also has no effect on the thermal conductivities of the composites. The electrical conductivities of the composites were significantly increased by more than $100 \%$. It will not be advisable to pretreat fibres with potassium permanganate where the composites are required to act as insulators.

\section{REFERENCES}

[1] M. Thirudutrambalam, A. Alavudeen, "Improving Mechanical Properties of Banana / Kenaf Polyester Hybrid Composites Using Sodium Lauryl Sulfate Treatments," Journal of Material Physics and Mechanics, vol. 8 p. 165-17, 2009.

[2] J. C.Bart, Additives in Polymers, Industrial Analysis and Applications, John Wiley, England. p. 14, 2005.

[3] M. Abdelmouleh, S. Bonfi, A. BenSalah, M. N. Belgacem, A. Gandini, "Modification of cellulose fibres with functionalized silane: effect of fibre treatment on the performance of cellulose - thermoset composites," J. Applied Polymer Science, vol. 98, pp. 974-984, 2005.

[4] M. S. Sreekala, M. D. Kumaran, G. Joseph, M. Jacob, S. Thomas, "Oil palm fibre phenol formaldehyde composites: Influence of fibre surface modification on the mechanical performance," Applied materials Composites, vol. 7 p. 295, 2000.

[5] S. Mohanty, S. K. Nayak, S. K. Verma, J. S. Tripathy, "Influence of fibre treatment on the performance of sisal-polypropylene composites," J. Reinforced Plastic Composite, vol. 23, pp. 625, 2005.

[6] S. Mishra, J. B. Naik, Y. P. Patil, "Reinforced Novalac Composites," Composite Science Technology, vol. 60, p. 1729, 2000.

[7] K. Joseph, L. H. C. Mattoso, R. D. Toledo, S. Thomas, "Natural Fibre Reinforced Composites," in Natural Polymers and Agrofibre Composites. E. Frorolini, A. L. Leao and L. H. C Matloso Eds, Sam Carlos Brazil Embrays US - IQSC UNESP pp. 159, 2000.

[8] A. Paul, K. Joseph, S. Thomas, "Effect of surface treatments on the electrical properties of low density polyethylene composites reinforced with short sisal fibre," Composite Science and Technology, vol. 57, pp. 67-79, 1997.

[9] K. C. M. Nair, S. Thomas, G. Groeninckx, "Mechanical properties of sisal fiber reinforced urea - formaldehyde," Composite Science Technology, vol. 61, pp. 2519, 2001. 